\title{
The General Form of non-Fock Coherent Boson States
}

\author{
By \\ Reinhard HONEGGER* and Alfred RIECKERs**
}

\begin{abstract}
Specifying their (normally ordered) characteristic functions we determine all states of the boson $C^{*}$-Weyl algebra which satisfy Glauber's coherence condition and are not realizable as density operators in Fock space. The pure ones are shown to be just the eigenstates of the annihilation operators in their GNS-representations (in contrast to the Fock case) and are characterized in many equivalent manners. The central decomposition of an arbitrary coherent state has the macroscopic phase variable as parameter and is supported by the pure coherent states, which is in fact the only way for a maximal decomposition. The set of all coherent states with the same absolute factorizing function is proven to be a Bauer simplex. The appearence of a classical coherent field part is studied in detail in the GNS-representations and shown to correspond to an enlargement of the set of one boson states by just one additional mode.
\end{abstract}

\section{§1. Introduction and Preliminary Results}

In one of his first papers on quantum optics [6] Glauber emphasizes the importance of states with a large number of photons for the description of light beams (and contrasts this with the few photon excitations in perturbational quantum electrodynamics). The relevant states, which have some degree of coherence, are investigated by him and his colleagues, however, only in the Fock representation. Since in laser beams there are in fact macroscopically many photons one may doubt if this formal limitation is justified. In [12] examples of fully coherent states are given, which cannot be represented by density operators in Fock space. A systematic study, where both Fock and non-Fock coherent states are investigated in the smeared field formalism, has been started recently in [13]. The aim of this paper is to carry on this analysis with the emphasis on non-Fock states.

We start here from Glauber's factorization condition for a (fully) coherent

Communicated by H. Araki, August 17, 1989.

* Institut für Theoretische Physik, Universität Tübingen, D-7400 Tübingen, Fed. Rep. of Germany.

** Institut für Theoretische Physik, Universität Tübingen, D-7400 Tübingen, Fed. Rep. of Germany. 
state [7]. If the creation and annihilation operators are smeared with oneboson test functions this coherence condition leads to a product of the values of a certain linear form on the test function space and its complex conjugate. In this formulation the consequences of the coherence condition can be deduced in a rigorous way, especially by means of the formalism of operator algebraic statistical mechanics [4]. If the mentioned linear form is bounded, then every state fulfilling this condition is given by a density operator in the Fock representation (i.e., is relatively normal to the Fock representation). Even in this more familiar case the complete classification of all coherent states seems not to have been carried through. If the mentioned linear form is unbounded the set of all (normalized mode) occupation numbers is unbounded and the corresponding state is no more representable by a density operator in Fock space (is disjoint to the Fock vacuum). In this case the typical operator algebraic techniques come into play. The statistical correlation inherent in the coherence condition extends now over so many photons (in states with finite particle density) that macroscopic classical features are generated, especially a macroscopic phase observable is displayed. Just these non-Fock structures seem to fit very well to many experimental situations with a high photon density. They are also in a certain analogy to the condensation phenomena of massive bosons, where in fact the "off-diagonal long-range order" is considered to be related to the coherence condition (cf. [11, p.39]). In the reconstructed quantum mechanics over these ordered states (by means of the GNSrepresentation) the collective phenomenon is concisely expressed by an additional classical field. Altogether there seems enough motivation for a thorough elaboration of the non-Fock coherent boson states.

In detail we proceed as follows. After preliminary results on states on the Weyl algebra we start in $\S 2$ with an analysis of the pure coherent states and refine the results of the literature. We use already here the technique of the Kolmogorov decomposition for positive-definite kernels (which is shortly introduced in the Appendix) in spite of its power being only visible in the case of mixed coherent states. The general form of coherent states is attacked by means of the (twofold) analytic power series of the normally ordered generating function. In spite of a quite different technique the result resembles that of [16]: the form of this series is fixed up to an infinite number of complex coefficients, which constitute an infinite positive complex matrix, where only the diagonal elements are determined by the coherence condition. The nondiagonal elements are here further analyzed for the non-Fock case, where one can show directly via the unboundedness of the mentioned linear form, that they depend solely on the difference of the integer matrix indices. This is in contrast to the Fock case, where the variety of admissible coefficients is larger. The Kolmogorov decomposition of the matrix coefficients leads to a Kolmogorov decomposition of the normally ordered generating function. 
In $\S 3$ we only treat the non-Fock case and show that the spectral measure of a certain unitary operator in the mentioned Kolmogorov decomposition leads to a unique decomposition of the given coherent state into pure coherent states and reveals the set of all coherent states with a fixed linear form to be a Bauer simplex. The GNS-representation is easily constructed and known in the literature for similar situations since [2]. Here it is gained by means of the minimal Kolmogorov decomposition of the characteristic function. It is always a tensor product of the Fock representation and the classical part. Especially elegant is the formulation of the represented Weyl algebra if the one-boson space is enlarged by an additional mode. This suggests also a natural enlargement of the $C^{*}$-Weyl algebra so that the classical field part may vary independently from the Fock part. The mathematical structure of this new $C^{*}$-Weyl algebra over a degenerate symplectic form is analyzed, a formalism which incorporates a whole classical statistics over the phase variable of the coherent field. This nontrivial classical part is shown to be approximable by local field expressions (without a classical component) in the strong resolvent sense.

Equipped with this mathematical machinery it is now easy to identify the decomposition of an arbitrary coherent state into pure coherent states as the central decomposition (which requires more than the pairwise disjointness of the supporting states). Moreover it is also shown to be the unique decomposition of a coherent state into a family of pure states (by means of a maximal measure not necessarily orthogonal). Related with this result is the fact that a coherent state can only be decomposed into countable many states if the latter are coherent with the same linear form as the given one, showing that the set of all coherent states with the same linear form is a face of the convex and compact state space of the original Weyl algebra.

Having anticipated some of the unique features of the non-Fock coherent states let us now start with the basic mathematical notions we shall need.

We denote by $E$ the pre-Hilbert space with (right linear) scalar product $\langle. \mid$.$\rangle , containing the genuine one-boson states which the free quantum particles$ may assume. (In certain situations it is physically reasonable to extend $E$, cf. e.g., §3.) Let $\mathscr{W}(E)$ be the unique $C^{*}$-algebra, which is generated by non-zero elements $W(f), f \in E$, satisfying the Weyl relations

$$
W(f)^{*}=W(-f), W(f) W(g)=\exp \left\{-\frac{i}{2} \mathfrak{J}\langle f \mid g\rangle\right\} W(f+g)
$$

for all $f, g \in E$. The states $\varphi$, which are elements of the weak*-compact convex state space $\mathscr{S}$ of $\mathscr{W}(E)$, are in bijective affine correspondence with the (characteristic) functions $C: E \rightarrow \mathbf{C}$ with $C(0)=1$ and for which the map $(f, q) \mapsto \exp \left\{\frac{i}{2} \mathfrak{J}\langle f \mid g\rangle\right\} C(g-f)$ constitutes a positive-definite kernel $E \times E$ $\rightarrow \mathbb{C}$ (cf. Appendix). If $C_{\varphi}$ is the characteristic function of $\varphi \in \mathscr{S}$, the minimal 
Kolmogorov decomposition of the associated positive-definite kernel is denoted by $V_{\varphi}: E \rightarrow \mathscr{H}_{\varphi}$, where $\mathscr{H}_{\varphi}$ is some Hilbert space. The set of all $C_{\varphi}, \varphi \in \mathscr{S}$, is denoted by $C(E)$.

If, e.g., $\varphi_{F}$ is the Fock vacuum state, then $C_{\varphi_{F}}(f) \equiv C_{F}(f)=\exp \left\{-\frac{1}{4}\|f\|^{2}\right\}$ and $V_{\varphi_{F}}(f) \equiv V_{F}(f)$ is the coherent vector $\Omega\left(\frac{i}{\sqrt{2}} f\right)$ in the Bose-Fock space $\mathscr{H}_{F}$ over $\bar{E}$, where $\bar{E}$ is the completion of $(E,\langle. \mid\rangle$.$) and$

$$
\Omega(f)=e^{-\frac{1}{2}\|f\|^{2}} \bigoplus_{n=0}^{\infty} \frac{1}{\sqrt{n !}}(f \otimes \cdots \otimes f)
$$

with the special case $\Omega(0)=\Omega_{F}$, the Fock vacuum vector. The Fock represented Weyl operators are characterized by the transformation property

$$
\prod_{F}(W(f)) \equiv W_{F}(f): \Omega(g) \mapsto \exp \left\{\frac{i}{\sqrt{2}} \Re\langle f \mid g\rangle\right\} \Omega\left(g+\frac{i}{\sqrt{2}} f\right), g, f \in E .
$$

A state $\varphi \in \mathscr{S}$ is called regular, if $C_{\varphi}(t f)$ is continuous in $t \in \mathbb{R}$ for all $f \in E$, and $\varphi$ is said to be of class $\mathscr{C}^{m}$, if $\mathbb{R} \ni t \mapsto C_{\varphi}(t f)$ is $m$-times differentiable for each $f \in E$. We set $\mathscr{C}^{\infty}:=\cap_{m \geq 1} \mathscr{C}^{m}$. $\quad \varphi$ is analytic, if for each $f \in E$ the map $t \mapsto C_{\varphi}(t f)$ may be extended in a complex neighborhood of $t=0$ to an analytic function $z \mapsto C_{\varphi}(z ; f)$. If for every $f \in E$ this neighborhood is all of $\mathbb{C}$, then $\varphi$ is called entire analytic. Especially $\varphi_{F}$ is entire analytic with $C_{F}(z ; f)=\exp \left\{-\frac{1}{4} z^{2}\|f\|^{2}\right\}$.

In the GNS-representation $\left(\mathbb{\Pi}_{\varphi}, H_{\varphi}, \Omega_{\varphi}\right)$ of a regular state $\varphi$ the boson field operator $\Phi_{\varphi}(f):=-\left.i \frac{d \Pi_{\varphi}(W(t f))}{d t}\right|_{t=0}$ is selfadjoint with domain $\mathscr{D}\left(\Phi_{\varphi}(f)\right)$ (given by the existence of the derivative), and the annihilation and creation operators

$$
a_{\varphi}(\mathrm{f})=\frac{1}{\sqrt{2}}\left(\Phi_{\varphi}(f)+i \Phi_{\varphi}(i f)\right), \quad a_{\varphi}^{*}(f)=\frac{1}{\sqrt{2}}\left(\Phi_{\varphi}(f)-i \Phi_{\varphi}(i f)\right)
$$

are closed on $\mathscr{D}\left(\Phi_{\varphi}(f)\right) \cap \mathscr{D}\left(\Phi_{\varphi}(i f)\right), a_{\varphi}^{*}(f)=a_{\varphi}(f)^{*}$ and $f \mapsto a_{\varphi}^{*}(f)$ is linear. If $P\left(\Phi_{\varphi}\right)$ symbolizes a polynomial in the field operators (depending on a finite selection of one-boson states), then we often write $\left\langle\varphi ; P\left(\Phi_{\varphi}\right)\right\rangle$ for $\left\langle\Omega_{\varphi} \mid P\left(\Phi_{\varphi}\right) \Omega_{\varphi}\right\rangle$, provided that $\Omega_{\varphi} \in \mathscr{D}\left(P\left(\Phi_{\varphi}\right)\right)$. The latter is the case for each polynomial with degree less or equal to $m$, if $\varphi$ is of class $C^{2 m}$. We mention, that the cyclic vector $\Omega_{\varphi}$ of the GNS-representation of $\varphi$ is an analytic (resp. entire analytic) vector for each $\Phi_{\varphi}(f), f \in E$, iff $\varphi$ is analytic (resp. entire analytic).

If $\varphi \in \mathscr{S}$ is analytic, for each $f \in E$ there is a neighborhood $U_{f}$ of the origin of the complex plane, such that 


$$
C_{\varphi}(z ; f)=\sum_{n=0}^{\infty} \frac{i^{n} z^{n}}{n !}\left\langle\varphi ; \Phi_{\varphi}(f)^{n}\right\rangle, z \in U_{f}
$$

In this case there is also a possibly smaller neighborhood $U_{f}^{\prime}$ of the origin with

$$
\ln C_{\varphi}(z ; f)=\sum_{n=0}^{\infty} \frac{i^{n} z^{n}}{n !}\left\langle\varphi ; \Phi_{\varphi}(f)^{n}\right\rangle_{\mathrm{T}}, \quad z \in U_{f}^{\prime}
$$

where the truncated expectation values $\langle\varphi ;\rangle_{\mathrm{T}}$ may be defined by this power series expansion of the left hand side (compare [4], Vol. II, p.40).

Lemma 1.1. Let $\varphi \in \mathscr{S} . \varphi$ is analytic, iff for each $f \in E$ there is a function $N_{\varphi}\left(z_{1}, z_{2} ; f\right)$, analytic in $U_{f} \times U_{f} \subseteq \mathbf{C}^{2}\left(U_{f}\right.$ a neighborhood of the origin of $\left.\mathbf{C}\right)$, such that

$$
C_{\varphi}(z f)=C_{F}(z f) N_{\varphi}(z, \bar{z} ; f) \forall z \in U_{f} .
$$

In this is the case, then it follows for the analytic extension of $\mathbf{R} \ni t \mapsto C_{\varphi}(t f)$

$$
\mathrm{C}_{\varphi}(z ; f)=C_{F}(z ; f) N_{\varphi}(z, z ; f) \forall z \in U_{f} .
$$

Especially $\varphi$ is entire analytic, iff $N_{\varphi}\left(z_{1}, z_{2} ; f\right)$ is entire analytic on $\mathbf{C}^{2}$ for every $f \in E$.

Moreover, the analyticity condition and (1.2) determine $N_{\varphi}$ uniquely to have the form

$$
\begin{aligned}
N_{\varphi}\left(z_{1}, z_{2} ; f\right) & \left.\left.=e^{\frac{1}{8}\left(z_{1}^{2}-z_{2}^{2}+2 z_{1} z_{2}\right)\|f\|^{2}}\left\langle e^{-\frac{i}{2}\left(\overline{z_{1}}+\overline{z_{2}}\right) \Phi_{\varphi}(f)} \Omega_{\varphi}\right| e^{-\frac{1}{2}\left(z_{2}-z_{1}\right.}\right)_{\varphi}(i f) \Omega_{\varphi}\right\rangle \\
& =\sum_{k, l=0}^{\infty}\left(\frac{i z_{1}}{\sqrt{2}}\right)^{k}\left(\frac{i z_{2}}{\sqrt{2}}\right)^{l} \frac{1}{k !} \frac{1}{l !}\left\langle\varphi ; a_{\varphi}^{*}(f)^{k} a_{\varphi}(f)^{l}\right\rangle, z_{1}, z_{2} \in U_{f} .
\end{aligned}
$$

Proof. Let $\varphi$ be analytic. Define $N_{\varphi}$ by equation (1.4). Then $N_{\varphi}$ is well defined and analytic in a neighborhood of the origin of $\mathrm{C}^{2}$, since $\Omega_{\varphi}$ is an analytic vecotr for $\Phi_{\varphi}(f)$ and $\Phi_{\varphi}(i f)$. Using the Weyl relations, one easily checks that (1.2) and (1.3) are fulfilled. Equation (1.5) is the Taylor series of (1.4), which one gets by use of the canonical commutation relations or the BakerHausdorff formula.

Conversely, if there exists an analytic function $N_{\varphi}(., ; f)$ satisfying (1.2), then it is unique by [3, p.36] and agrees with the one defined by (1.4).

A state $\varphi$ is called quasi-free, if it is analytic and $\left\langle\varphi ; \Phi_{\varphi}(f)^{n}\right\rangle_{\mathrm{T}}=0$ for $n>2$ and all $f \in E$. One concludes from (1.1) that in this case

$$
C_{\varphi}(f)=\exp \left\{i\left\langle\varphi ; \Phi_{\varphi}(f)\right\rangle-\frac{1}{2}\left\langle\varphi ; \Delta \Phi_{\varphi}(f)^{2}\right\rangle\right\},
$$

where 


$$
\left\langle\varphi ; \Delta \Phi_{\varphi}(f)^{2}\right\rangle=\left\langle\varphi ; \Phi_{\varphi}(f)^{2}\right\rangle-\left\langle\varphi ; \Phi_{\varphi}(f)\right\rangle^{2}=\left\langle\varphi ; \Phi_{\varphi}(f)^{2}\right\rangle_{\mathrm{T}} .
$$

Definition 1.2. A state $\omega \in \mathscr{S}$ is called coherent, if it is of class $\mathscr{C}^{\infty}$ and if there is a linear form $L: E \rightarrow \mathbf{C}$ such that

$$
\left\langle\omega ; a_{\omega}^{*}\left(f_{1}\right) \cdots a_{\omega}^{*}\left(f_{k}\right) a_{\omega}\left(g_{1}\right) \cdots a_{\omega}\left(g_{l}\right)\right\rangle=L\left(f_{1}\right) \cdots L\left(f_{k}\right) \overline{L\left(g_{1}\right)} \cdots \overline{L\left(g_{l}\right)}
$$

for all $k, l \in N_{0}$ with $k=l$ and all $f_{1}, \ldots, f_{k}, g_{1}, \ldots, g_{l} \in E$. The set of all coherent states with linear form $L$ is denoted by $\mathscr{S}_{L}$.

From (1.6) it follows that $\mathscr{S}_{L}$ is convex (the extreme boundary of which is denoted by $\left.\partial_{e} \mathscr{S}_{L}[1]\right)$. If $\omega \in \mathscr{S}_{L}$, then also $\omega \in \mathscr{S}_{z L}$ for all elements $z$ of the torus

$$
\mathrm{T}:=\{w \in \mathbf{C}|| w \mid=1\}
$$

\section{§ 2. Series Expansions and Kolmogorov Decompositions for the Characteristic Functions}

The most common states in quantum optics, which satisfy the coherence condition, fulfill (1.6) even for all $k, l \in \mathbf{N}_{0}$. We first analyze this class of states in the smeared field formalism and demonstrate, beside other things, that in fact all choices of the linear form $L$ lead to well defined states on the Weyl algebra $\mathscr{W}(E)$. The following results extend Theorem 2.1 of [13].

Proposition 2.1 Let $L: E \rightarrow \mathbb{C}$ be an arbitrary linear form. It follows:

(a) The function $C_{L}: E \rightarrow \mathbb{C}$, given by

$$
C_{L}(f)=C_{F}(f) \exp \{i \sqrt{2} \mathfrak{R} L(f)\}, f \in E,
$$

is in $C(E)$ and defines an entire analytic state $\varphi_{L}$ with (cf. Lemma 1.1)

$$
N_{L}\left(z_{1}, z_{2} ; f\right)=\exp \left\{\frac{i}{\sqrt{2}}\left(z_{1} L(f)+z_{2} \overline{L(f)}\right)\right\} \forall z_{1}, z_{2} \in \mathbb{C} .
$$

The minimal Kolmogorov decomposition of the associated positive-definite kernel is of the form

$$
V_{L}(f):=V_{F}(f) \exp \{i \sqrt{2} \mathfrak{R} L(f)\} \in \mathscr{H}_{F},
$$

where-as in Section 1-the index " $F$ " refers to the Fock vacuum.

(b) For $\varphi \in \mathscr{S}$ the following conditions are equivalent:

(i) $\varphi=\varphi_{L}$;

(ii) $\varphi$ is of class $\mathscr{C}^{\infty}$ and satisfies (1.6) for all $k, l \in \mathbf{N}_{0}$;

(iii) $\varphi$ is of class $\mathscr{C}^{2}$ and satisfies $(1.6)$ for $(k, l)=(0,1)$ and $(k, l)=(1.1)$ for all $f=g$; 
(iv) $\varphi$ is regular and satisfies $a_{\varphi}(f) \Omega_{\varphi}=\overline{L(f)} \Omega_{\varphi} \forall f \in E$ in its GNSrepresentation.

(c) For $\varphi \in \mathscr{S}$ is equivalent:

(i) $\varphi=\varphi_{z L}$ for some $z \in \mathrm{T}$;

(ii) $\varphi \in \mathscr{S}_{L}$ and $\varphi$ is quasi-free.

(d) $A \varphi \in \mathscr{S}$ with

(i ) $\varphi=\varphi_{z L}$ for some $z \in T$

satisfies also

(ii) $\varphi \in \mathscr{S}_{L}$ and $\varphi$ is pure.

It then (trivially) fulfills

(iii) $\varphi \in \mathscr{S}_{L}$ and $\varphi$ is primary;

(iv) $\varphi \in \partial_{e} \mathscr{S}_{L}$;

(v) $\varphi$ is in the support of a maximal measure (on $\mathscr{S}$ ), which decomposes an $\omega \in \mathscr{S}_{L}$.

Remark. For unbounded $L$ all the conditions of (d), and thus of (c), are equivalent to those of (b), if therein $L$ is replaced by $z L, z \in T$ (cf. Proposition 3.6 (a)).

Proof. (a) $C_{L}$ is the product of $C_{F} \in C(E)$ and a positive-definite function on $E$, which leads to $C_{L} \in C(E)$ and the stated minimal Kolmogorov decomposition. Obviously $N_{L}\left(z_{1}, z_{2} ; f\right)$ is entire analytic on $\mathbb{C}^{2}$ and gives the analytic extension $C_{L}(z ; f)=C_{F}(z ; f) N_{L}(z, z ; f)$, as well as $C_{L}(z f)=C_{F}(z f) N_{L}(z$, $\bar{z} ; f)$ (cf. Lemma 1.1).

(b)(ii) $\Rightarrow$ (iii) and (iv) $\Rightarrow$ (ii) are trivial. (i) $\Rightarrow$ (iii) is obtained by calculating the derivatives $\left.\frac{\partial^{k+l} N_{L}\left(z_{1}, z_{2} ; f\right)}{\partial z_{1}^{k} \partial z_{2}^{l}}\right|_{z_{1}=z_{2}=0}$ for $(k, l)=(0,1)$ and $(k, l)=(1,1)$ and comparing this with (1.5). (iii) $\Rightarrow\left(\right.$ iv): One calculates directly $\left\|\left(a_{\varphi}(f)-\lambda\right) \Omega_{\varphi}\right\|^{2}$ $=|\overline{L(f)}-\lambda|^{2}$, which is zero for $\lambda=\overline{L(f)}$. (iv) $\Rightarrow$ (i) one gets by use of (1.2) and (1.5) with $z=1: C_{\varphi}(f)=C_{F}(f) e^{i \sqrt{2 \Re L(f)}}$.

(c) (i) $\Rightarrow$ (ii): Comparing $C_{z L}(f)$ according to (2.1) with (1.1) we find

$$
\left\langle\varphi ; \Phi_{\varphi}(f)\right\rangle=\sqrt{2} \mathfrak{R}(z L(f)) \text { and }\left\langle\varphi ; \Delta \Phi_{\varphi}(f)^{2}\right\rangle=\frac{1}{2}\|f\|^{2}
$$

and the higher truncated functionals vanishing, thus $\varphi_{z L}$ is quasi-free. Coherence follows from (b)(ii). The reverse implication is given in the Appendix of [13].

(d) (i) $\Rightarrow$ (ii): From (a) and the Appendix we conclude that the Weyl operators in the GNS-representation of $\varphi_{z L}$ are scalar multiples of the FockWeyl operators and hence generate an irreducible $C^{*}$-algebra and thus $\varphi_{z L}$ is pure. (ii) $\Rightarrow$ (iii) and (ii) $\Rightarrow$ (iv) are immediate. (ii) $\Rightarrow$ (v) is gained by taking for $\omega$ the pure state $\varphi_{z L}$. 
As a preparatory step for he general case let us consider condition (1.6) for $k=l=1$.

Lemma 2.2. Let $\omega \in \mathscr{S}$ be of class $\mathscr{C}^{2}$, then the following two conditions are equivalent :

(i) $\left|\left\langle\omega ; a_{\omega}^{*}(f) a_{\omega}(g)\right\rangle\right|=\left\|a_{\omega}(f) \Omega_{\omega}\right\|\left\|a_{\omega}(g) \Omega_{\omega}\right\|$ for all $f, g \in E$;

(ii) there exists a linear form $L: E \rightarrow \mathbb{C}$ such that $\left\langle\omega ; a_{\omega}^{*}(f) a_{\omega}(g)\right\rangle=$ $L(f) \overline{L(g)}$ for all $f, g \in E$.

Moreover, if these conditions are satisfied, then

$$
\overline{L(g)} a_{\omega}(f) \Omega_{\omega}=\overline{L(f)} a_{\omega}(g) \Omega_{\omega} \quad \forall f, g \in E .
$$

The linear form $L$ is determined uniquely by (ii), up to a phase.

Proof. (i) $\Rightarrow$ (ii): If $\omega=\varphi_{F}$ this is trivially valid with $L \equiv 0$. If $\omega \neq \varphi_{F}$, there exists a $h \in E$ with $\left\|a_{\omega}(h) \Omega_{\omega}\right\|=1$. Choose $L(f):=\left\langle\omega ; a_{\omega}^{*}(f) a_{\omega}(h)\right\rangle$. One easily checks $\left\|\left(a_{\omega}(f)-\overline{L(f)} a_{\omega}(h)\right) \Omega_{\omega}\right\|^{2}=0 \forall f \in E$, from which one gets $a_{\omega}(f) \Omega_{\omega}$ $=\overline{L(f)} a_{\omega}(h) \Omega_{\omega} . \quad$ Now (ii) and $\overline{L(g)} a_{\omega}(f) \Omega_{\omega}=\overline{L(f)} a_{\omega}(g) \Omega_{\omega}$ are immediate.

(ii) $\Rightarrow$ (i) follows directly since $\left\|a_{\omega}(f) \Omega_{\omega}\right\|^{2}=\left\langle\omega ; a_{\omega}^{*}(f) a_{\omega}(f)\right\rangle=|L(f)|^{2}$.

Let $\omega$ be of class $\mathscr{C}^{\infty}$ and satisfying the conditions of Lemma 2.2 for some linear form $L$ (first order coherence [16]). If we choose for $L \neq 0$ an $h \in E$ such that $L(h)=1$ (iff $L=0$ then $\omega=\varphi_{F}$ ), then by Lemma $2.2 a_{\omega}(f) \Omega_{\omega}=\overline{L(f)}$ $a_{\omega}(h) \Omega_{\omega} \forall f \in E$. Consequently

$$
\begin{aligned}
& \left\langle\omega ; a_{\omega}^{*}\left(f_{1}\right) \cdots a_{\omega}^{*}\left(f_{k}\right) a_{\omega}\left(g_{1}\right) \cdots a_{\omega}(g l)\right\rangle= \\
& \quad=L\left(f_{1}\right) \cdots L\left(f_{k}\right) \overline{L\left(g_{1}\right)} \cdots \overline{L\left(g_{l}\right)}\left\langle\omega ; a_{\omega}^{*}(h)^{k} a_{\omega}(h)^{l}\right\rangle .
\end{aligned}
$$

Hence for a state $\omega \in \mathscr{S}$ to be in $\mathscr{S}_{L}$ it is necessary and sufficient that $\omega$ is of class $\mathscr{C}^{\infty}$ and

$\left\langle\omega ; a_{\omega}^{*}(f) a_{\omega}(g)\right\rangle=L(f) \overline{L(g)}$ for all $f, g \in E$ and

- $\left\langle\omega ; a_{\omega}^{*}(h)^{n} a_{\omega}(h)^{n}\right\rangle=|L(h)|^{2 n}$ for some $h \notin \operatorname{ker}(L)$ for each $n \geq 2$.

Proposition 2.3. Let be $\omega \in \mathscr{S}$ and $L$ an arbitrary linear form on $E$. Then it is equivalent:

(i) $\omega \in \mathscr{S}_{L}$;

(ii) there exists a family $c(k, l) \in \mathbb{C}, k, l \in \mathbb{N}_{0}$, which constitutes a positive 
definite kernel on $\mathbf{N}_{0} \times \mathbf{N}_{0}$ with $c(k, k)=1 \forall k \in \mathbf{N}_{0}$ such that for each $f \in E$

$$
C_{\omega}(f)=C_{F}(f) \sum_{k, l=0}^{\infty}\left(\frac{i}{\sqrt{2}}\right)^{k+l} \frac{1}{k !} \frac{1}{l !} L(f)^{k} \overline{L(f)} l c(k, l) .
$$

Furtheron, the properties of the $c(k, l)$ imply $\overline{c(k, l)}=c(l, k)$ and $|c(k, l)| \leq 1$ for all $k, l \in \mathbb{N}_{0}$, showing $\omega$ to be entire analytic.

Proof. (i) $\Rightarrow$ (ii): Let $\omega \in \mathscr{S}_{L}$. Use equation (2.2) and set

$$
c(k, l):=\left\langle\omega ; a_{\omega}^{*}(h)^{k} a_{\omega}(h)^{l}\right\rangle \quad \forall k, l \in \mathbb{N}_{0},
$$

from which also the positive-definiteness of the kernel $c(k, l)$ follows. Letting $n$ $=2$ in equation (A.1) of the Appendix, we see that the matrix $\left(\begin{array}{ccc}c(k, k) c(k, l) \\ c(l, k) c(l, l)\end{array}\right)$ must be positive and therefore selfadjoint with positive determinant. As $\omega$ is coherent we have $\mathrm{c}(k, k)=1=c(l, l)$, which implies $\overline{c(k, l)}=c(l, k)$ and $\mid c(k$, $l) \mid \leq 1$ for all $k, l \in \mathbf{N}_{0}$. Consequently, inserting (2.2) in (1.5), the convergence of the series $N_{\omega}\left(z_{1}, z_{2} ; f\right)$ is absolute for each $z_{1}, z_{2} \in \mathbf{C}$ and from (1.2) we obtain (2.3) by setting $z=1$. By Lemma $1.1 \omega$ is entire analytic. (ii) $\Rightarrow$ (i): Obviously $C_{\omega}(z f)=C_{F}(z f) \sum_{k, l=0}^{\infty}\left(\frac{i}{\sqrt{2}}\right)^{k+l} \frac{1}{k !} \frac{1}{l !} z^{k} \bar{z}^{l} L(f)^{k} \overline{L(f)} l c(k, l)$ for all $z \in \mathbb{C}$ and $f \in E$, where the double series converges absolutely because of $|c(k, l)| \leq 1$. Hence with (1.2) and (1.5) one gets $\left\langle\omega ; a_{\omega}^{*}(f)^{k} a_{\omega}(f)^{l}\right\rangle=L(f)^{k} \overline{L(f)}{ }^{l} c(k, l)$. Now use $c(1.1)=1$ and the polarization identity to verify $\left\langle\omega ; a_{\omega}^{*}(f) a_{\omega}(g)\right\rangle=L(f) \overline{L(g)} \forall f$, $g \in E$, which implies (2.2) for an $h \in E$ with $L(h)=1$. For this $h(2.4)$ is then again valid, which leads with $c(k, k)=1 \forall k \in \mathbf{N}_{0}$ to the coherence condition (Definition 1.2).

We now give the cases in which $L$ is unbounded a special treatment, where often the following simple fact is employed for arguments, which go back to [2].

Lemma 2.4. If $L: E \rightarrow \mathbb{C}$ is unbounded, then there is for every $\alpha \in \mathbb{C} a$ sequence $\left(f_{n}^{\alpha}\right)_{n \in \mathbf{N}}$ in $E$ with

$$
\lim _{n \rightarrow \infty}\left\|f_{n}^{\alpha}\right\|=0 \text { and } \lim _{n \rightarrow \infty} L\left(f_{n}^{\alpha}\right)=\alpha .
$$

Proof. Since Lis unbounded, there is a sequence $\left(f_{n}\right)_{n \in \mathbf{N}}$ in $E$ with $\left\|f_{n}\right\|=1$ and $\lim _{n \rightarrow \infty} L\left(f_{n}\right)=\infty$. Without restriction in generality $L\left(f_{n}\right) \neq 0 \forall n$, and we may define $f_{n}^{\alpha}:=\frac{\alpha f_{n}}{L\left(f_{n}\right)}$. 
Proposition 2.5. A coherent state $\omega \in \mathscr{S}_{L}$ is normal to the Fock representation, iff $L$ is bounded.

Proof. (a) Let $L$ be bounded. By (1.5) and Proposition 2.3 the functions $E \ni f \mapsto N_{\omega}\left(z_{1}, z_{2}, f\right)$ are continuous for all $z_{1}, z_{2} \in \mathbb{C}$ and hence $\omega$ extends canonically to $\mathscr{W}(\bar{E})$. Moreover, there is a $h \in \bar{E}$ so that $L(f)=\langle h \mid f\rangle$ $\forall f \in E$. Then $\bar{E}=E_{1} \oplus E_{2}$ with $E_{1}=\mathrm{Ch}$. From (2.3) one concludes that $\omega$ $=\omega_{1} \otimes \omega_{2}$ is a product state on $\mathscr{W}(\bar{E})=\mathscr{W}\left(E_{1}\right) \otimes \mathscr{W}\left(E_{2}\right)$, where $\omega_{1}$ is normal to the Fock representation of $\mathscr{W}\left(E_{1}\right)$-since $E_{1}$ is finite-dimensional [4, Corollary 5.2.15] - and $\omega_{2}$ is the Fock vacuum on $\mathscr{W}\left(E_{2}\right)$.

(b) If $L$ is unbounded, we find from equation (2.3) with the sequence of Lemma 2.4

$$
\lim _{n \rightarrow \infty} C_{\omega}\left(f_{n}^{\alpha}\right)=\sum_{k, l=0}^{\infty}\left(\frac{i}{\sqrt{2}}\right)^{k+l} \frac{1}{k !} \frac{1}{l !} \alpha^{k} \bar{\alpha}^{l} c(k, l) \quad \forall \alpha \in \mathbb{C} .
$$

Since $c(k, k) \neq 0 \forall k \in \mathbf{N}_{0}$, the values (2.5) are not constant in $\alpha$ (which is seen by an analytic extension to $\mathbb{C}^{2}$ and [3, p.36]). If on the other hand there would be a density operator $\rho_{\omega}$ on $\mathscr{H}_{F}$ which represents $\omega$, then

$$
\lim _{n \rightarrow \infty} C_{\omega}\left(f_{n}^{\alpha}\right)=\lim _{n \rightarrow \infty} \operatorname{tr}_{\mathscr{H}_{F}}\left\{\rho_{\omega} W_{F}\left(f_{n}^{\alpha}\right)\right\}=1 \quad \forall \alpha \in \mathbb{C}
$$

since by $\left[4\right.$, Proposition 5.2.4] $W_{F}():. E \rightarrow \mathscr{B}\left(\mathscr{H}_{F}\right)$ is normrespectively strong operator continuous. To avoid this contradiction $\omega$ cannot be normal to the Fock representation.

For unbounded $L$ we now show that the kernel $c(k, l)$ of Proposition 2.3 only depends on the difference $k-l$.

Theorem 2.6 For a given linear form $L: E \rightarrow \mathbb{C}$ consider the following conditions on the function $C: E \rightarrow \mathbb{C}$ :

(a) $C=C_{\omega}$ for some $\omega \in \mathscr{S}_{L}$;

(b) there exists a positive-definite function $d: \mathbb{Z} \rightarrow \mathbb{C}$ with $d(0)=1$ such that for all $f \in E$

$$
C(f)=C_{F}(f) \sum_{k, l=0}^{\infty}\left(\frac{i}{\sqrt{2}}\right)^{k+l} \frac{1}{k !} \frac{1}{l !} L(f)^{k} \overline{L(f)}^{l} d(k-l)
$$

(c) there exists a Hilbert space $\mathscr{H}_{v}$, a unitary $v$ acting on $\mathscr{H}_{v}$ and a normalized $w \in \mathscr{H}_{v}$ such that for all $f \in E$

$$
C(f)=C_{F}(f) N(f) \quad \forall f \in E
$$

where $N($.$) is a positive-definite function on the additive group E$ with a Kolmogorov decomposition 


$$
Y(f)=\exp \left\{\frac{i}{\sqrt{2}}\left(L(f) v+\overline{L(f)} v^{*}\right)\right\} w .
$$

Then it holds (a) $\Rightarrow(b)$ if $L$ is unbounded, whereas $(\mathrm{b}) \Leftrightarrow(\mathrm{c}) \Rightarrow(\mathrm{a})$ is valid for arbitrary $L$.

Proof. (a) $\Rightarrow$ (b) for unbounded $L$ : From the equations (2.2) and (2.4) we get for $f \notin \operatorname{ker}(L)$ and $l \geq k$

$$
\left\langle\omega ; a_{\omega}^{*}(f)^{k} a_{\omega}(f)^{l}\right\rangle=L(f)^{k} \overline{L(f)}^{l} c(k, l),\left\langle\omega ; a_{\omega}(f)^{l-k}\right\rangle=\overline{L(f)}^{l-k} c(0, l-k) .
$$

Hence

$$
\begin{aligned}
& \left|L(f)^{k} \overline{L(f)}^{l}(c(k, l)-c(0, l-k))\right|= \\
& \quad=\left|\left\langle\left(a_{\omega}^{*}(f)^{k} a_{\omega}(f)^{k}-|L(f)|^{2 k}\right) \Omega_{\omega} \mid a_{\omega}(f)^{l-k} \Omega_{\omega}\right\rangle\right| \\
& \quad \leq\left\|\left(a_{\omega}^{*}(f)^{k} a_{\omega}(f)^{k}-|L(f)|^{2 k}\right) \Omega_{\omega}\right\|\left\|a_{\omega}(f)^{l-k} \Omega_{\omega}\right\| .
\end{aligned}
$$

Here we have by the canonical commutation relations and Definition 1.2

$$
\begin{aligned}
& \left\|\left(a_{\omega}^{*}(f)^{k} a_{\omega}(f)^{k}-|L(f)|^{2 k}\right) \Omega_{\omega}\right\|^{2}= \\
& =\sum_{r=1}^{k} q_{r}\|f\|^{2 r}\left\langle\Omega_{\omega} \mid a_{\omega}^{*}(f)^{2 k-r} a_{\omega}(f)^{2 k-r} \Omega_{\omega}\right\rangle+ \\
& \quad+\left\langle\Omega_{\omega} \mid a_{\omega}^{*}(f)^{2 k} a_{\omega}(f)^{2 k} \Omega_{\omega}\right\rangle-2|L(f)|^{2 k}\left\langle\Omega_{\omega} \mid a_{\omega}^{*}(f)^{k} a_{\omega}(f)^{k} \Omega_{\omega}\right\rangle+|L(f)|^{4 k} \\
& =\sum_{r=1}^{k} q_{r}\|f\|^{2 r}|L(f)|^{2(2 k-r)}
\end{aligned}
$$

for certain $q_{r} \in \mathbf{N}_{0}$ with $q_{r} \neq 0$ at least for one $r$. Observing this and $\left\|a_{\omega}(f)^{l-k} \Omega_{\omega}\right\|=|L(f)|^{l-k}$ in (2.9) yields

$$
|c(k, l)-c(0, l-k)|^{2} \leq \sum_{r=1}^{k} q_{r}\left(\frac{\|f\|}{|L(f)|}\right)^{2 r} .
$$

Using the sequence $\left(f_{n}^{\alpha}\right)_{n \in \mathbf{N}}$ of Lemma 2.4 with $\alpha \neq 0$ gives $c(k, l)=c(0, l-k)$ for $l \geq k$. If $l<k$ then $c(k, l)=\overline{c(l, k)}=\overline{c(0, k-l)}=c(k-l, 0)$. Defining

$$
d(m):= \begin{cases}c(m, 0) & \text { if } m \geq 0 \\ c(0,-m) & \text { if } m<0\end{cases}
$$

gives $d(k-l)=c(k, l) \forall k, l \in \mathbf{N}_{0}$ and transforms (1.2) and (1.5) with (2.3) into (2.6), where $d(0)=c(k, k)=1$ by the coherence definition.

(b) $\Rightarrow$ (c) for arbitrary L: Applying for $d$ the Kolmogorov decomposition for 
positive-definite functions on groups (cf. Appendix) one obtains $d(k-l)=$ $\left\langle v^{l} w \mid v^{k} w\right\rangle, w \in \mathscr{H}_{v}$ and $v$ an unitary on $\mathscr{H}_{v}$ for some Hilbert space $\mathscr{H}_{v}$. Inserting this into (2.6), where $f$ gets replaced by $g-f$, leads to $N(g-f)$ $=\langle Y(f) \mid Y(g)\rangle$ with $Y$ given by $(2.8)$ and the exponential is definable by a power series converging in the operator norm. Thus $N$ is a positive-definite function on $E$ and $1=d(0)=\|w\|^{2}=\|Y(0)\|^{2}=N(0)$.

(c) $\Rightarrow$ (b) and (c) $\Rightarrow$ (a) for arbitrary $L: C(f):=C_{F}(f) N(f)$ is normalized and $(f, g) \mapsto \exp \left\{\frac{i}{2} \mathfrak{I}\langle f \mid g\rangle\right\} C(g-f)$ a positive-definite kernel because of the product property. Thus $C \in C(E)$ and therefore defines a state $\omega \in \mathscr{S}$. Now, using $N(f)$ $=\langle Y(0) \mid Y(f)\rangle$ we see that it has the form (2.3) with specialized $c(k, l)$ $=\left\langle v^{l} w \mid v^{k} w\right\rangle=\left\langle w \mid v^{k-l} w\right\rangle:=d(k-l)$ from which $c(k, k)=\|w\|^{2}=d(0)=1$ follows.

From the above proof it is obvious, that $k \mapsto v^{k} w$ is a minimal Kolmogorov decomposition of $d$, iff $Y$ of (2.8) is so for $N$.

For unbounded linear forms $L: E \rightarrow \mathbb{C}$, Theorem 2.6 solves the classification problem for coherent states completely. We shall see in the next section that in this case $\frac{1}{\sqrt{2}}\left(L(f) v+\overline{L(f)} v^{*}\right)$ is the classical part of the field operator $\Phi_{\omega}(f)$ in the GNS-representation of the coherent state $\omega$.

\section{§3. Integral Decompositions and GNS-Representations}

In this section we stick to the assumption that the linear form $L: E \rightarrow \mathbb{C}$, occurring in the coherence definition (Definition 1.2), is unbounded.

The one-dimensional torus $T$ (cf.(1.7)) is a compact abelian group, the dual (character) group of $\mathbb{Z}(\mathbb{Z}$ equipped with the discrete topology). $T$ acts on $\mathscr{W}(E)$ by means of the ${ }^{*}$-automorphisms (the gauge transformations of the first kind)

$$
\tau_{z}(W(f))=W(z f), z \in \top
$$

Since $\|\left(\tau_{z}-1\right)(W(f))=2$ for all $z \neq 1$ and $f \neq 0$, the action of these gauge transformations in the Heisenberg picture is not pointwise norm continuous. By means of the dual transformations $\tau_{z}^{*}(\varphi):=\varphi \circ \tau_{z}, \varphi \in \mathscr{S}, z \in T$ (Schrödinger picture) we define for given linear form $L$ the mapping

$$
j_{L}: \top \longrightarrow \mathscr{S}, z \longrightarrow \tau_{z}^{*}\left(\varphi_{L}\right)=: j_{L}(z),
$$

where $\varphi_{L}$ is the pure coherent state of Proposition 2.1 (a). From the characteristic functions $C_{z L}$ (cf.(2.1)) we see that $j_{L}$ is a weak*-continuous injection. The set $M_{+}^{1}(T)$ of all probability measures on $T$ is a Bauer simplex, 
since $T$ is a compact Hausdorff space [1, Corollary II.4.2]. The extreme boundary $\partial_{e} M_{+}^{1}(T)$ may be identified with $T$, since it consists of the point measures on $T$. For later use let us introduce for every $\alpha \in \mathbf{C}$ the function

$$
g_{\alpha}: \top \longrightarrow \mathrm{C}, z \longmapsto \exp \{i \sqrt{2} \mathfrak{R}(z \alpha)\}
$$

Obviously $g_{\alpha} \in C(T)$, the continuous functions on $T$. In fact $L H\left\{g_{\alpha} \mid \alpha \in \mathbf{C}\right\}$ is a subalgebra of $C(T)$, which is invariant under complex conjugation and separates points of $T$; thus it is norm dense in $C(T)$ by means of the Stone-Weierstraß theorem [14, Theorem IV.10].

Theorem 3.1. Let $L: E \rightarrow \mathbf{C}$ be an unbounded linear form. It follows, that there is a one-to-one correspondence between coherent states $\omega \in \mathscr{S}_{L}$ and probability measures $\mu \in M_{+}^{1}(T)$ such that

$$
\omega=\int_{T} \tau_{z}^{*}\left(\varphi_{L}\right) d \mu(z) .
$$

Moreover, $\mathscr{S}_{L}$ is a Bauer simplex with extreme boundary

$$
\partial_{e} \mathscr{S}_{L}=\left\{\tau_{z}^{*}\left(\varphi_{L}\right) \mid z \in T\right\}=\left\{\varphi_{Z L} \mid z \in T\right\} .
$$

Proof. (a) For $\omega \in \mathscr{S}_{L}$ consider in Theorem 2.6 equation (2.8) the spectral decomposition $v=\int_{T} z d E_{v}(z)$ and obtain $N(f)=\langle Y(0) \mid Y(f)\rangle=\mu\left(g_{L(f)}\right)$, where $d \mu(z)=\left\langle w \mid d E_{v}(z) w\right\rangle$. Then by (2.7) and the gauge invariance of $C_{F}(f)$ one gets $C_{\omega}(f)=\int_{T} C_{L}(z f) d \mu(z)$. Since the affine bijection between states and characteristic functions associates $\tau_{z}^{*}\left(\varphi_{L}\right)$ with $C_{L}(z f)$, the validity of (3.2) follows.

(b) Let (3.2) be valid for some $\mu \in M_{+}^{1}(T)$. Define on $E$ the positivedefinite function $N(f):=\mu\left(g_{L(f)}\right)$. If $\mathscr{H}_{v}:=L^{2}(T, \mu)$ and $(v f)(z):=z f(z)$ and $w(z) \equiv 1$, then $Y(f)$ defined by $(2.8)$ is a (minimal) Kolmogorov decomposition of $N$. Hence equation (2.7) of Theorem 2.6 defines a coherent state $\omega \in \mathscr{S}_{L}$.

Since $L H\left\{g_{\alpha} \mid \alpha \in \mathbf{C}\right\}$ is dense in $C(T)$ two measures $\mu, \rho$ agree, iff $\mu\left(g_{\alpha}\right)$ $=\rho\left(g_{\alpha}\right) \forall \alpha$ and from $\langle\omega ; W(f)\rangle=C_{F}(f) \mu\left(g_{L(f)}\right)$ follows the uniqueness.

(c) The bijection from $M_{+}^{1}(\top)$ onto $\mathscr{S}_{L}$ given by (3.2) is also affine and vague-weak*-continuous: $\mu_{i} \stackrel{i \in I}{\longrightarrow} \mu$ in the vague topology of $M_{+}^{1}(T)$ is equivalent to $\mu_{i}\left(g_{\alpha}\right) \stackrel{i \in I}{\longrightarrow} \mu\left(g_{\alpha}\right) \forall \alpha \in \mathbf{C}$. Thus, for the associated states $\omega_{i}$ and $\omega$ we have

$$
C_{\omega_{l}}(f)=C_{F}(f) \mu_{i}\left(g_{L(f)}\right) \stackrel{i \in I}{\longrightarrow} C_{F}(f) \mu\left(g_{L(f)}\right)=C_{\omega}(f) \quad \forall f \in E,
$$

which gives $\omega_{i} \stackrel{i \in I}{\longrightarrow} \omega$ in the weak*-topology. Hence $\mathscr{S}_{L}$ is a Bauer simplex affinely isomorphic to $M_{+}^{1}(\top)$ with $\partial_{e} \mathscr{S}_{L}$ given by the images of the point measures in $M_{+}^{1}(\top)$, which coincide with $j_{L}(z), z \in T$.

Of course, it also would have been possible to use Bochner's theorem for the positive-definite function $d$ of Theorem 2.6 to get the integral decomposition 
(3.2). But in the above proof, via the spectral decomposition of the unitary $v$, we have indirectly shown the equivalence of the Kolmogorov decomposition and the decomposition due to Bochner's theorem for the $d(k-l)=\int_{T} z^{k-l} d \mu(z)$ $=\left\langle v^{l} w \mid v^{k} w\right\rangle$. Iff the Kolmogorov decomposition of $d$ is minimal, the support of the corresponding measure $\mu \in M_{+}^{1}(T)$ agrees with the spectrum of $v$.

Proposition 3.2. Let $L$ be unbounded. For $\omega \in \mathscr{S}_{L}$ the minimal Kolmogorov decomposition $V_{\omega}$ (associated with the kernel $\left.\exp \left\{\frac{i}{2} \mathfrak{J}\langle f \mid g\rangle\right\} C_{\omega}(g-f)\right)$ has the form

$$
V_{\omega}(f)=V_{F}(f) \otimes Y_{\omega}(f) \in \mathscr{H}_{F} \otimes \mathscr{H}_{v}
$$

where $Y \equiv Y_{\omega}$ given in (2.8) is choosen minimal. If $W_{v}(\alpha):=\exp \left\{\frac{i}{\sqrt{2}}(\alpha v\right.$ $\left.\left.+\bar{\alpha} v^{*}\right)\right\}, \alpha \in \mathbb{C}$, for the GNS-representation of $\omega$ we have

$$
\mathscr{H}_{\omega}=\mathscr{H}_{F} \otimes \mathscr{H}_{v}, \quad \Omega_{\omega}=\Omega_{F} \otimes w
$$

and

$$
\Pi_{\omega}(W(f))=W_{F}(f) \otimes W_{v}(L(f)) \quad \forall f \in E,
$$

which is not continuous in $f \in E$ with respect to the strong operator topology. If $\mathscr{M}_{v}$ is the $W^{*}$-algebra generated by the $W_{v}(\alpha)$ in $\mathscr{H}_{v}$ then

$$
\begin{aligned}
& \mathscr{M}_{\omega}:=\Pi_{\omega}(\mathscr{W}(E))^{\prime \prime}=\mathscr{B}\left(\mathscr{H}_{F}\right) \bar{\otimes} \mathscr{M}_{v} \\
& \mathscr{M}_{\omega}^{\prime}=\Pi_{\omega}(\mathscr{W}(E))^{\prime}=1_{F} \otimes \mathscr{M}_{v},
\end{aligned}
$$

and the center is

$$
\mathscr{Z}_{\omega}:=\mathscr{M}_{\omega} \cap \mathscr{M}_{\omega}^{\prime}=1_{F} \otimes \mathscr{M}_{v}
$$

where $\bar{\otimes}$ denotes the $W^{*}$-tensor product introduced in [15].

Proof. For $\left(f_{n}^{\alpha}\right)_{n \in \mathbf{N}}$ of Lemma 2.4 we get for all $\alpha \in \mathbb{C}$

$$
s-\lim _{n \rightarrow \infty} W_{F}\left(f_{n}^{\alpha}\right) \otimes W_{v}\left(L\left(f_{n}^{\alpha}\right)\right)=1_{F} \otimes W_{v}(\alpha),
$$

where the strong operator continuity of $W_{F}():. E \rightarrow \mathscr{B}\left(\mathscr{H}_{F}\right)$ [4, Proposition 5.2.4] and that of $W_{v}():. \mathbb{C} \rightarrow \mathscr{B}\left(\mathscr{H}_{v}\right)$ has been used. Thus $\Pi_{\omega}(W()$.$) is not strong$ operator continuous. From (3.3) and the cyclicity of $w$ we conclude that $\Omega_{F} \otimes \mathscr{H}_{v} \subseteq \overline{L H}\left\{V_{\omega}(f) \mid f \in E\right\}$. Since $\left[W_{F}(f) \otimes W_{v}(L(f))\right]\left[1_{F} \otimes W_{v}(-L(f))\right]$ $\Omega_{F} \otimes w=W_{F}(f) \Omega_{F} \otimes w$ we also find $\mathscr{H}_{F} \otimes w \subseteq \overline{L H}\left\{V_{\omega}(f) \mid f \in E\right\}$, which leads 
to $\mathscr{H}_{F} \otimes \mathscr{H}_{v}=\overline{L H}\left\{V_{\omega}(f) \mid f \in E\right\} . \quad$ Now it is easy to check that $\left(\Pi_{\omega}, \mathscr{H}_{\omega}, \Omega_{\omega}\right)$ is the GNS-representation of $\omega$. The rest follows again from (3.3) and the fact that $\mathscr{M}_{v}$ is a maximal abelian von Neumann algebra on $\mathscr{H}_{v}$ (having the cyclic vector w) and from [15, Chapter IV].

The discontinuity of $\Pi_{\omega}(W()):. E \rightarrow \mathscr{B}\left(\mathscr{H}_{\omega}\right)$ for $\omega \in \mathscr{S}_{L}(L$ unbounded) may be overcome by modifying $E$ and defining a new $C^{*}$-Weyl algebra.

Let us first equip $E$ with the new scalar product $\langle f \mid g\rangle_{L}:=\langle f \mid g\rangle$ $+\overline{L(f)} L(g), f, g \in E$. This suggests to work with pairs $\xi:=f \oplus \alpha \in \bar{E} \oplus \mathbf{C}$ $=: \overline{\mathscr{K}}(\bar{E}$ is the completion of $E$ with respect to the old scalar product $\langle. \mid\rangle$.$) and$ to embed $E$ therein by means of

$$
\lambda: E \longrightarrow \overline{\mathscr{K}}, f \longmapsto \lambda(f):=f \oplus L(f) .
$$

Since the natural scalar product in $\overline{\mathscr{K}}$ is $\langle\xi \mid \eta\rangle_{\mathscr{K}}=\langle f \mid g\rangle+\bar{\alpha} \beta$ for $\xi=f \oplus \alpha$ and $\eta=g \oplus \beta$ we have $\langle\lambda(f) \mid \lambda(g)\rangle_{\mathscr{K}}=\langle f \mid g\rangle_{L} \forall f, g \in E$ and for the sequence $\left(f_{n}^{\alpha}\right)_{n \in \mathbf{N}}$ of Lemma 2.4 holds $\lim _{n \rightarrow \infty}\left\|\lambda\left(f_{n}^{\alpha}\right)-0 \oplus \alpha\right\|_{\mathscr{K}}=0$, which shows that $\overline{\mathscr{K}}$ is the completion of $E$ with respect to $\langle. \mid\rangle_{L}$. Because of $\|\lambda(f)\|_{K} \geq\|f\| \forall f \in E$ the inverse map $\lambda^{-1}: \lambda(E) \rightarrow E$ extends to a contraction $\chi: \overline{\mathscr{K}} \rightarrow \bar{E}$.

In view of an extension of the Weyl algebra $\mathscr{W}(E)$ we first introduce the nondegenerate symplectic form $\sigma$ on $\lambda(E)$ by $\sigma(\lambda(f), \lambda(g)): \mathfrak{J}\langle f \mid g\rangle \forall f, g \in E$, which is bounded since $|\sigma(\xi, \eta)| \leq|\langle\chi(f) \mid \chi(g)\rangle| \leq\|\xi\|_{\mathscr{K}}\left\|_{\eta}\right\|_{\mathscr{K}} \forall \xi, \eta \in \lambda(E)$. Denote by $\sigma_{\mathscr{K}}$ the continuous extension of $\sigma$ to all of $\overline{\mathscr{K}}$

Lemma 3.3. It is $\chi(f \oplus \alpha)=f \forall f \in \bar{E}, \alpha \in \mathbf{C}$ and

$$
\mathscr{K}_{s}:=\operatorname{ker}(\chi)=\left\{\xi \in \overline{\mathscr{K}} \mid \sigma_{\mathscr{K}}(\xi, \eta)=0 \forall_{\eta} \in \mathscr{K}\right\}=0 \oplus \mathbf{C} .
$$

Moreover, the positive quadratic form $E \times E \ni(f, g) \mapsto \overline{L(f)} L(g)$ is singular (with respect to $\langle. \mid\rangle$.$) and the minimal singular subspace is \mathscr{K}_{s}$.

Proof. Let $f \oplus \alpha \in \overline{\mathscr{K}}$ and $\left(f_{n}\right)_{n \in \mathbf{N}}$ a sequence in $E$ with $\lim _{n \rightarrow \infty} \| \lambda\left(f_{n}\right)$ $-f \oplus \alpha \|_{\mathscr{K}}=0$. Then $\lim _{n \rightarrow \infty}\left\|f_{n}-f\right\|=0$ and hence by the continuity of $\chi$ one gets $\chi(f \oplus \alpha)=\lim _{n \rightarrow \infty} \chi\left(\lambda\left(f_{n}\right)\right)=\lim _{n \rightarrow \infty} f_{n}=f$. By a similar approximation proce- 
dure we obtain $\sigma_{\mathscr{K}}(\mathrm{f} \oplus \alpha, g \oplus \beta)=\mathfrak{J}\langle f \mid g\rangle$ from which $\operatorname{ker}\left(\sigma_{\mathscr{K}}\right)=\operatorname{ker}(\chi)$ $=0 \oplus \mathbb{C}$ follows. Since $\chi$ restricted to $\overline{\mathscr{K}} \ominus \mathscr{K}_{s}$ is an unitary onto $\bar{E}$ the form $\overline{L(.)} L($.$) is purely singular. The latter concept is part of a theory described in$ [8].

Define $\mathscr{K}:=E \oplus \mathbb{C}$. We want to extend $\mathscr{W}(E)$ to a Weyl algebra over this pre-Hilbert space $\mathscr{K}$. Since $\sigma_{\mathscr{K}}$ is degenerate on $\mathscr{K}$ there is no canonical way to associate a $C^{*}$-Weyl algebra with it, and we do this in a special way in the GNSrepresentations of the coherent states $\omega \in \mathscr{S}_{L}$. For certain purposes $\mathscr{W}(E)$ may be even extended to a Weyl algebra over $\overline{\mathscr{K}}$.

Proposition 3.4 For $\omega \in \mathscr{S}_{L}$ (L unbounded) consider the GNS-representation of Proposition 3.2 and define for $\xi=f \oplus \alpha \in \overline{\mathscr{K}}$

$$
W_{\omega}(\xi):=W_{F}(f) \otimes W_{v}(\alpha) \in \mathscr{M}_{\omega}=\Pi_{\omega}(\mathscr{W}(E))^{\prime \prime} .
$$

If $\mathscr{A}_{v}$ denotes the $C^{*}$-algebra generated by the $W_{v}(\alpha), \alpha \in \mathbb{C}$, then it holds

( i ) $W_{\omega}(\lambda(f))=\Pi_{\omega}(W(f))$, for all $f \in E$;

(ii ) $W_{\omega}(\xi)=W_{\omega}\left(\xi^{\prime}\right)$, iff $f=f^{\prime}$ and $g_{\alpha}(z)=g_{\alpha}^{\prime}(z)$ for $\mu$-almost all $z \in T$, where $\mu$ is the measure associated with $\omega$ according to Theorem 3.1;

(iii) $W_{\omega}(\xi) W_{\omega}(\eta)=\exp \left\{-\frac{i}{2} \sigma_{\mathscr{K}}(\xi, \eta)\right\} W_{\omega}(\xi+\eta)$ and $W_{\omega}(\xi)^{*}=W(-\xi)$ for all $\xi, \eta \in \overline{\mathscr{K}}$

(iv ) $\left\langle\Omega_{\omega} \mid W_{\omega}(\xi) \Omega_{\omega}\right\rangle=C_{F}(f) \mu\left(g_{\alpha}\right)$;

(v) $W_{\omega}():. \overline{\mathscr{K}} \rightarrow \mathscr{M}_{\omega}$ is continuous in the strong operator topology;

( vi ) $\overline{L H}\left\{W_{\omega}(\xi) \mid \xi \in \mathscr{K}\right\}=\Pi_{F}(\mathscr{W}(E)) \otimes \mathscr{A}_{v}$, where $\mathscr{A}_{v}$ is the $C^{*}$-algebra generated by the $W_{v}(\alpha), \alpha \in \mathbb{C}\left(\right.$ the $C^{*}$-tensor product is unique, $\mathscr{A}_{v}$ being abelian [15, Chapter IV]);

(vii) $\overline{L H}\left\{W_{\omega}(\xi) \mid \xi \in \overline{\mathscr{K}}\right\}=\Pi_{F}(\mathscr{W}(\bar{E})) \otimes \mathscr{A}_{v}$;

(viii) $\overline{L H}^{s}\left\{W_{\omega}(\xi) \mid \xi \in \mathscr{K}_{s}\right\}=\mathscr{Z}_{\omega}=\mathscr{M}_{\omega}^{\prime}$ (the closure in the strong operator topology);

(ix ) Let $\Psi_{\omega}(\xi)$ resp. $\Phi_{\omega}(f)$ generate the unitary groups $\left\{W_{\omega}(t \xi) ; t \in \mathbb{R}\right\}$ resp. $\left\{\Pi_{\omega}(W(t f)) ; t \in \mathbb{R}\right\}$, where $\xi \in \overline{\mathscr{K}}$ and $f \in E$, and let $\Phi_{v}(\alpha):=$ $\frac{1}{\sqrt{2}}\left(\alpha v+\bar{\alpha} v^{*}\right), \alpha \in \mathbf{C} . \quad$ Then $\overline{\mathscr{K}} \ni \xi=f \oplus \alpha \mapsto \Psi_{\omega}(\xi)=\Phi_{F}(f) \otimes 1_{v}$ $+1_{F} \otimes \Phi_{v}(\alpha)$ is continuous in the strong resolvent sense and $\Phi_{\omega}(f)$ 
$=\Psi_{\omega}(\lambda(f)), f \in E$, approximates therefore $\Psi_{\omega}(\xi)$ for all $\xi \in \overline{\mathscr{K}}$.

Proof. (i) follows from Proposition 3.2. (ii): The Fock parts are equal, iff $f=f^{\prime}$. From $\left\|\left(W_{v}(\alpha)-W_{v}(\beta)\right) W_{v}(\gamma) w\right\|^{2}=2-2 \Re\left\langle g_{\alpha} \mid g_{\beta}\right\rangle_{\mu}$ and the cyclicity of $\omega$ one gets $W_{v}(\alpha)=W_{v}(\beta)$, iff $g_{\alpha}=g_{\beta} \mu$-almost everywhere. (iii) and (iv) follow by direct calculation and (v) by the strong operator continuity of $E \ni f \mapsto W_{F}(f)$ and $C \ni \alpha \mapsto W_{v}(\alpha)$. (vi) and (vii) one finds directly from the definition of the $W_{\omega}(\xi)$ and (viii) is immediate from Proposition 3.2 and $W_{\omega}(0 \oplus \alpha)=1_{F} \otimes W_{v}(\alpha)$. (ix) follows from (i) and (v).

If $s_{\mu}=\operatorname{supp}(\mu)$ is the support of the measure $\mu \in M_{+}^{1}(T)$ associated as before with $\omega \in \mathscr{S}_{L}$, then by the spectral mapping theorem [9, Theorem 4.4 .5 and Example 2.4.11] we have

$$
\mathscr{A}_{v} \cong \mathscr{C}\left(s_{\mu}\right), \mathscr{H}_{v} \cong L^{2}\left(s_{\mu}, \mu\right), \mathscr{M}_{v} \cong L^{\infty}\left(s_{\mu}, \mu\right),
$$

where $d \mu(z)=\left\langle w \mid d E_{v}(z) w\right\rangle, z \in \top$ with $v=\int_{\top} z d E_{v}(z)$ and $W_{v}(\alpha)$ gets identified with $g_{\alpha} \mid s_{\mu}$ restricted to $s_{\mu}$.

For some more insight into the structure of the extended Weyl algebra of Proposition 3.4 we give the following remark. In [10] there is defined a $C^{*}$ Weyl algebra $\overline{\Delta(H, \sigma)}$ over a (possibly degenerate) symplectic space $(H, \sigma)$ by means of the completion with the maximal $C^{*}$-norm of the *-algebra $\Delta(H, \sigma)$, which in turn is algebraically generated by functions $\delta_{x}$ on $\left.H\right) \delta_{x}(x)=1$ and $\delta_{x}(y)$ $=0$ elsewhere) with the product $\delta_{x} \cdot \delta_{y}=e^{-\frac{i}{2} \sigma(x, y)} \delta_{x+y}$ and involution $\delta_{x}^{*}$ $=\delta_{-x}$. If there is another $C^{*}$-norm $\|\cdot\|_{0}$ on $\Delta(H, \sigma)$, there exists a closed $*_{-}$ ideal $\mathscr{T}$ in $\overline{\Delta(H, \sigma)}$ such that one has the quotient structure $\overline{\Delta(H, \sigma)}^{0}$ $=\overline{\Delta(H, \sigma)} / \mathscr{T}$.

Now, if $\omega=\int_{T} \tau_{z}^{*}\left(\varphi_{L}\right) d \mu(z)$ this gives rise to the symplectic space $\left(\mathscr{K}, \sigma_{K}\right)$ and the corresponding $C^{*}$-Weyl algebra $\overline{\Delta\left(\mathscr{K}, \sigma_{\mathscr{K}}\right)}$. With $\tilde{C}(f \oplus \alpha):=C_{F}(f)$ $\mu\left(g_{\alpha}\right)$ is associated a positive-definite kernel $\mathscr{K} \times \mathscr{K} \ni(\xi, \eta) \mapsto \exp \left\{\frac{i}{2} \sigma_{\mathscr{K}}(\xi, \eta)\right\}$ $\widetilde{C}(\eta-\xi)$, which by [10] defines a state $\tilde{\omega}$ on $\overline{\Delta\left(\mathscr{K}, \sigma_{\mathscr{K}}\right)}$ with $\tilde{\omega}\left(\delta_{\xi}\right)$ $=\widetilde{C}(\xi) \forall \xi \in \mathscr{K}$. Obviously $\tilde{\omega}$ is an extension of $\omega$, its GNS-representation is given by $\mathscr{H}_{\varpi}=\mathscr{H}_{\omega}, \Omega_{\varpi}=\Omega_{\omega}$ and $\Pi_{\tilde{\omega}}\left(\delta_{\xi}\right)=W_{\omega}(\xi), \xi \in \mathscr{K}$. The representation $\Pi_{\varpi}$ defines a $C^{*}$-or a $C^{*}$-half-norm on $\Delta\left(\mathscr{K}, \sigma_{\mathscr{K}}\right)$, the completion of which is $\mathscr{W}(E) \otimes \mathscr{C}\left(s_{\mu}\right)$, the $C^{*}$-algebra of Proposition 3.4 (vi). If $\mu$ is the Haar measure on $T$, then one gets $\mathscr{W}(E) \otimes \mathscr{C}(T)$. Each $\omega \in \mathscr{S}_{L}$ can be extended to an $\hat{\omega}$ on $\mathscr{W}(E) \otimes \mathscr{C}(\top) \quad$ so that $\hat{\omega}\left(W(f) \otimes g_{\alpha}\right)=C_{F}(f) \mu\left(g_{\alpha}\right)$ and the algebras $\mathscr{W}(E) \otimes \mathscr{C}\left(s_{\mu}\right)$ can be obtained as the quotient of $\mathscr{W}(E) \otimes \mathscr{C}(T)$ with the closed *-ideal $\mathscr{W}(E) \otimes \mathscr{C}_{\infty}\left(T \backslash s_{\mu}\right)\left(C_{\infty}(X)\right.$ denotes the continuous functions vanishing at infinity). 
In order to analyze further the decomposition of $\omega \in \mathscr{S}_{L}$ by means of the measure $\mu \in M_{+}^{1}(T)$, which is described in Theorem 3.1, let us transfer $\mu$ to a regular Borel measure $\mu_{\omega} \in M_{+}^{1}(\mathscr{S})$ on the state space $\mathscr{S}$ (equipped with the weak*-topology) by setting

$$
\mu_{\omega}(B):=\mu\left(j_{L}^{-1}(B)\right) \text { for each Borel set } B \subseteq \mathscr{S},
$$

where $j_{L}$ is from (3.1). Since $T$ is compact and $j_{L}$ continuous, $j_{L}(T)=\partial_{e} \mathscr{S}_{L}$ is weak*-compact an $\mu_{\omega}$ is supported by $\partial_{e} \mathscr{S}_{L}$. Every $\mu$-measurable function $h: T \rightarrow \mathbb{C}$ is transferred to a $\mu_{\omega}$-measurable function $\hat{h}: \mathscr{S} \rightarrow \mathbb{C}$ by setting $\hat{h}(\varphi)$ $=0$ if $\varphi \notin \partial_{e} \mathscr{S}_{L}$ and $\hat{h}(\varphi)=h(z)$ if $\varphi=\varphi_{z L}, z \in T$. In this way $L^{p}(T, \mu)$ and $L^{p}\left(\mathscr{S}, \mu_{\omega}\right)$ can be identified for every $p \in[1, \infty]$.

Proposition 3.5. For $\omega \in \mathscr{S}_{L}$ (L unbounded) the measure $\mu_{\omega}$ of (3.2) and (3.4) is its central measure.

Proof. The Tomita map $k_{\omega}: L^{\infty}\left(\mathscr{S}, \mu_{\omega}\right) \rightarrow \mathscr{M}_{\omega}^{\prime}$ for $\mu_{\omega} \in M_{+}^{1}(\mathscr{S})$ is uniquely defined by (cf. [4, Lemma 4.1.21])

$$
\left\langle\Omega_{\omega} \mid k_{\omega}(F) \Pi_{\omega}(A) \Omega_{\omega}\right\rangle=\int_{\mathscr{S}} F(\varphi)\langle\varphi: A\rangle d \mu_{\omega}(\varphi) \forall F \in L^{\infty}\left(\mathscr{S}, \mu_{\omega}\right) \forall A \in \mathscr{W}(E) .
$$

For any $f \in E$ and $\alpha \in \mathbb{C}$ we calculate

$$
\begin{aligned}
\left\langle\Omega_{\omega} \mid W_{\omega}(0 \oplus \alpha) \Pi_{\omega}(W(f)) \Omega_{\omega}\right\rangle & =\left\langle\Omega_{\omega} \mid W_{F}(f) \otimes W_{v}(L(f)+\alpha) \Omega_{\omega}\right\rangle \\
& =C_{F}(f) \mu\left(g_{L(f)+\alpha}\right) \\
& =\int_{T} g_{\alpha}(z)\left\langle\varphi_{z L} ; W(f)\right\rangle d \mu(z) \\
& =\int_{\mathscr{S}} \widehat{g_{\alpha}}(\varphi)\langle\varphi ; W(f)\rangle d \mu_{\omega}(\varphi) .
\end{aligned}
$$

Replacing $W(f)$ by linear combinations of Weyl operators and performing limits in the norm of $\mathscr{W}(E)$ we obtain

$$
k_{\omega}\left(\widehat{g_{\alpha}}\right)=W_{\omega}(0 \oplus \alpha) \quad \forall \alpha \in \mathbb{C} .
$$

Obviously $k_{\omega}$ is a *-homomorphism on $N_{L}:=L H\left\{\widehat{g_{\alpha}} \mid \alpha \in \mathbb{C}\right\}$. Since $N_{L}$ is $\sigma\left(L^{\infty}, L^{1}\right)$-dense in $L^{\infty}\left(\mathscr{S}, \mu_{\omega}\right)$ we conclude from the continuity property of $k_{\omega}$ [4, Lemma 4.1.21] that it is a *homomorphism from $L^{\infty}\left(\mathscr{S}, \mu_{\omega}\right)$ onto the smallest abelian von Neumann algebra in $\Pi_{\omega}(\mathscr{W}(E))^{\prime}$ which contains all $W_{\omega}(0 \oplus \alpha)$, $\alpha \in \mathbb{C}$. Now the assertion follows from [4, Proposition 4.1.22] and Proposition 3.4 (viii).

If $E$ is separabel, each state $\varphi=\varphi_{z L} \in \operatorname{supp}\left(\mu_{\omega}\right) \subseteq \partial_{e} \mathscr{S}_{L}$ has the GNSrepresentation $\left(\Pi_{\varphi}, \mathscr{H}_{F}, \Omega_{F}\right)$ with $\Pi_{\varphi}(W(f))=\exp \{i \sqrt{2} \mathfrak{R}(z L(f))\} W_{F}(f)$ and 
separable Fock space $\mathscr{H}_{F}$. Then the direct integral exists and by Effros' theorem [4, Theorem 4.4.9] we get a spatial decomposition of the GNSrepresentation of $\omega$

$$
\left(\Pi_{\omega}, \mathscr{H}_{\omega}, \Omega_{\omega}\right)=\int_{\mathscr{S}}^{\oplus}\left(\Pi_{\varphi}, \mathscr{H}_{\varphi}, \Omega_{\varphi}\right) d \mu_{\omega}(\varphi) .
$$

Let us still draw some further conclusions from the described decomposition of coherent states.

Proposition 3.6. Let $L$ be unbounded. Then:

(a) For each $\omega \in \mathscr{S}_{L}$ there is a unique maximal measure $\mu \in M_{+}^{1}(\mathscr{S})$ such that $\omega=\int_{\partial_{e} S} \varphi d \mu(\varphi)$, namely the central measure $\mu=\mu_{\omega}$. Moreover, in Proposition $2.1($ d) we have the implications (i) $\Leftrightarrow(\mathrm{ii}) \Leftrightarrow(\mathrm{iii}) \Leftrightarrow(\mathrm{iv}) \Leftrightarrow(\mathrm{v})$.

(b) $\mathscr{S}_{L}$ is a face of $\mathscr{S}$.

Proof. (a) follows from $\mathscr{M}_{\omega}^{\prime}=\mathscr{Z}_{\omega}$ and [15, Lemma IV. 6.26] and the fact that the only pure and only primary states of $\mathscr{S}_{L}$ are in $\partial_{e} \mathscr{S}_{L}=\left\{\varphi_{z L} \mid z \in T\right\}$.

(b) If $\omega=\lambda \varphi_{1}+(1-\lambda) \varphi_{2}, \omega \in \mathscr{S}_{L}, \varphi_{1}, \varphi_{2} \in \mathscr{S}$ and $0<\lambda<1$, then find two maximal measures $\mu_{1}, \mu_{2} \in M_{+}^{1}(\mathscr{S})$ decomposing $\varphi_{1}$ and $\varphi_{2}$ (see e.g. [4, Proposition 4.1.3] for the existence). Then $\mu:=\lambda \mu_{1}+(1-\lambda) \mu_{2}$ is a maximal measure (cf. [4, Proposition 4.1.14]), which decomposes $\omega$, and is equal to the central measure according to the reasoning in (a). Consequently $\operatorname{supp}\left(\mu_{k}\right) \subseteq$ $\partial_{e} \mathscr{S}_{L}$ for $k \in\{1,2\}$, and therefore $\varphi_{1}, \varphi_{2} \in \mathscr{S}_{L}$.

Altogether the foregoing mathematical analysis provides a detailed picture how a classical collective structure arises from the coherence condition with unbounded linear form $L$. For all such $L$ 's there shows up exactly one macroscopically occupied mode with a classical (central) phase observable. Giving this phase fluctuation free values is the only way to decompose the given coherent state into pure quantum states. If the considered bosons are photons then classical fields in the GNS-representations give an indication, how classical optics may be founded by quantum optics, a view which is also supported by the examples in [12].

\section{Acknowledgments}

The authors profited from discussions with A. Rapp, who inspired several arguments by his own work on the subject. One of us (R. H.) is grateful to the Deutsche Forschungsgemeinschaft for financial support.

\section{Appendix}

We collect here some basic notions and facts on positive-definite kernels and 
apply this to the characteristic functions of states on the Weyl algebra.

Restricting the definition of [5] to the complex valued kernels we call a mapping $K: X \times X \rightarrow \mathbb{C}$ a positive-definite kernel, if for all $n \in \mathbb{N}$, all $x_{1}, \ldots, x_{n} \in X, X$ being an arbitrary set, and all $z_{1}, \ldots, z_{n} \in \mathbb{C}$

$$
\sum_{i, j=1}^{n} \bar{z}_{i} z_{j} K\left(x_{i}, x_{j}\right) \geq 0 .
$$

A Kolmogorov decomposition of the positive-definite kernel $K$ is defined to be a mapping $V: X \rightarrow \mathscr{H}_{V}$, where $\mathscr{H}_{V}$ is a (not further specified) Hilbert space, such that

$$
K(x, y)=\langle V(x) \mid V(y)\rangle \quad \forall x, y \in X
$$

On the other hand (A.2) implies positive-definiteness of the kernel $K$. A Kolmogorov decomposition is called minimal, if

$$
\mathscr{H}_{V}=\overline{L H}\{V(x) \mid x \in X\}
$$

For every positive-definite kernel there exists a minimal Kolmogorov decomposition. If $V: X \rightarrow \mathscr{H}_{V}$ and $V^{\prime}: \mathscr{H}_{V^{\prime}}$ are Kolmogorov decompositions of $K$ and $V$ is minimal, then there is a unique isometry $U: \mathscr{H}_{V} \rightarrow \mathscr{H}_{V^{\prime}}$, such that $V^{\prime}(x)=U V(x) \forall x \in X$. If $V^{\prime}$ is also minimal, then $U$ is unitary.

Let be $\mathscr{G}$ a group, then $T: \mathscr{G} \rightarrow \mathbb{C}$ is called a positive-definite function, if $K(g$, $\left.g^{\prime}\right):=T\left(g^{-1} g^{\prime}\right)$ constitutes a positive-definite kernel $\mathscr{G} \times \mathscr{G} \rightarrow \mathbb{C}$. In this case the Kolmogorov decompositions of $K$ are given by vectors $V(g)=\mathbb{I}(g) w \in \mathscr{H}_{V}$, where $w \in \mathscr{H}_{V}$ and $\Pi$ is a unitary representation of $\mathscr{G}$ on $\mathscr{H}_{V}$.

If $K_{1 / 2}: X \times X \rightarrow \mathbb{C}$ are two positive-definite kernels with Kolmogorov decompositions $V_{1 / 2}: X \rightarrow \mathscr{H}_{1 / 2}$, then $X \ni x \mapsto V_{1}(x) \otimes V_{2}(x)$ gives a Kolmogorov decomposition of the positive-definite kernel $K_{1}(x, y) K_{2}(x, y): X \times X$ $\rightarrow \mathbb{C}$. It is in general not minimal, even if the $V_{1 / 2}$ are so.

For every state $\varphi \in \mathscr{S}$ on the Weyl algebra $\mathscr{W}(E)$ with the characteristic function $C_{\varphi}: E \rightarrow \mathbb{C}$, the mapping $E \times E \ni(f, g) \mapsto \exp \left\{\frac{i}{2} \mathfrak{J}\langle f \mid g\rangle\right\} C_{\varphi}(g-f)$ is a positive-definite kernel. If $V_{\varphi}: E \rightarrow \mathscr{H}_{\varphi}$ is the corresponding minimal Kolmogorov decomposition, then one checks immediately (compare $[5, \mathrm{p} .44])$ that $V_{\varphi}(\cdot$ $+g) \exp \left\{\frac{i}{2} \mathfrak{J}\langle\cdot \mid g\rangle\right\}$ is also one. Thus the mappings $W_{\varphi}(g): V_{\varphi}(f) \mapsto V_{\varphi}(f$ $+g) \exp \left\{\frac{i}{2} \mathfrak{I}\langle f \mid g\rangle\right\}$ extend to a unique family of unitaries on $\mathscr{H}_{\varphi}$, which satisfy the Weyl commutation relations, and by (A.3) is cyclic with respect to the vector $\Omega_{\varphi}:=V_{\varphi}(0)$. By Slawny's theorem (the uniqueness of the Weyl algebra up to *isomorphism) and since $\left\langle\Omega_{\varphi} \mid W_{\varphi}(f) \Omega_{\varphi}\right\rangle=C_{\varphi}(f)$ we have a realization of the 
GNS-representation $\left(\Pi_{\varphi}, \mathscr{H}_{\varphi}, \Omega_{\varphi}\right)$ of $\mathscr{W}(E)$ corresponding to $\varphi$, and $\Pi_{\varphi}(W(f))$ $=W_{\varphi}(f) \forall f \in E$. This shows that to every function $C$ in $C(E)$ (defined in the Introduction) there corresponds a unique state $\varphi \in \mathscr{S}$, which is constructively given by the cyclic vector in the minimal Kolmogorov decomposition of $C$.

\section{References}

[1] Alfsen, E. M., Compact Convex Sets and Boundary Integrals, (Springer Verlag, Berlin, Heidelberg, New York, 1971).

[2] Araki, H., Woods, E. J., Representations of the Canonical Commutation Relations Describing a Nonrelativistic Infinite Free Bose Gas, J. Math. Phys., 5 (1963), 637-662.

[3] Bochner, S., Martin, W. T., Several Complex Variables, (Princeton University Press, 1948).

[4] Bratteli, O., Robinson, D. W., Operator Algebras and Quantum Statistical Mechanics, Vol. I, II, (Springer Verlag, Berlin, Heidelberg, New York, 1979, 1981).

[5] Evans, D. E., Lewis, J. T., Dilations of Irreversible Evolutions in Algebraic Quantum Theory, Communications of the Dublin Institute for Advanced Studies, Series A (Theoretical Physics), 24 (1977).

[6] Glauber, R. J., Coherent and Incoherent States of the Radiation Field, Phys. Rev., 131 (1963), 2766-2788.

[7] - The Quantum Theory of Optical Coherence, Phys. Rev., 130 (1963), 2529-2539.

[8] Honegger, R., Decompostion of Positive Sesquilinear Forms and the Central Decomposition of Gauge-Invariant Quasi-Free States on the Weyl-Algebra, Z. Natürforsch., A45 (1990), 1728.

[9] Kadison, R. V., Ringrose, J. R., Fundamentals of the Theory of Operator Algebras, Vol. I, II, (Academic Press, New York, London, Toronto, Sydney, San Francisco, 1983, 1986).

[10] Manuceau, J., Sirugue, M., Testard, D., Verbeure, A., The Smallest $C^{*}$-Algebra for Canonical Commutation Relations, Commun math. Phys., 32 (1973), 231-243.

[11] Nussenzveig, H. M., Introduction to Quantum Optics, (Gordon and Breach Verlag, London, New York, Paris, 1973).

[12] Provost, J. P., Rocca, F., Vallee, G., Sirugue, M., Phase Properties of some Photon States with Nonzero Energy Density, J. Math. Phys., 15 (1974), 2079-2085.

[13] Rapp, A., General Glauber Coherent States on the Weyl Algebra and their Phase Integrals, (Preprint, Tübingen, 1989).

[14] Reed, M., Simon, B., Methods of Modern Mathematical Physics, Vol. I, Functional Analysis, (Academic Press, New York, London, Toronto, Sydney, San Francisco, 1980).

[15] Takesaki, M., Theory of Operator Algebras, Vol. I, (Springer Verlag, Berlin, Heidelberg, New York, 1979).

[16] Titulaer, U. M., Glauber, R. J., Density Operators for Coherent Fields, Phys. Rev., 145 (1966), 1041-1050. 
